\title{
THE CONVEX HULL PROPERTY OF IMMERSED MANIFOLDS
}

\author{
ROBERT OSSERMAN
}

Let $M_{0}$ be an $m$-dimensional differentiable manifold, and let $M$ be defined by an immersion $f: M_{0} \rightarrow \boldsymbol{R}^{n}$. We shall say that $M$ has the convex hull property if, for every domain $D$ on $M_{0}$ such that $f$ maps $D$ into a bounded set in $\boldsymbol{R}^{n}$, the image of $D$ lies in the convex hull of its boundary values. It is well known that minimal submanifolds of $\boldsymbol{R}^{n}$ have this property. The usual proof of this uses the fact that the coordinate functions in $\boldsymbol{R}^{n}$ are harmonic functions on $M$ if $M$ is minimal. (See, for example, Lemma 7.1 in [1] for the case $m=2, n$ arbitrary. We may note that the proof there is not quite correct as it stands, since the assumption that the image lies in a bounded set is implicitly used but not stated.)

Our purpose here is to give a simple geometric condition characterizing those manifolds having the convex hull property.

Theorem. A manifold $M$ has the convex hull property if and only if, at each point of $M$, there does not exist any normal direction with respect to which all normal curvatures of $M$ are positive.

Remarks. 1. If the normal curvatures with respect to a normal $N$ are all negative, then those with respect to $-N$ are all positive. Thus the condition of the theorem is equivalent to the property that, for each normal $N$, the range of values of the normal curvatures with respect to $N$ includes zero. If we denote the principal curvatures with respect to $N$ in decreasing order by

$$
k_{1}(N) \geq k_{2}(N) \geq \cdots \geq k_{m}(N)
$$

then another equivalent formulation is

$$
k_{1}(N) k_{m}(N) \leq 0 \quad \text { for every normal } N .
$$

Thus for surfaces in $\boldsymbol{R}^{3}$, the condition of the theorem is simply that the Gauss curvature be everywhere nonpositive.

2. If $M$ is minimal, then at each point,

$$
k_{1}(N)+\cdots+k_{m}(N)=0 \quad \text { for every normal } N
$$

Received April 24, 1971. This work was partially supported by a grant from NSF. 
Since clearly $(2) \Rightarrow(1)$, the result for minimal manifolds is an immediate consequence of the theorem.

3. The fact that condition (1) is not only a sufficient condition for the convex hull property but also a necessary one was pointed out to the author by David Hoffman. A further remark of Hoffman's is that, for an arbitrary manifold, condition (2) holds for any normal $N$ orthogonal to the mean curvature vector, and hence the same is true of (1).

4. If, in the definition of the convex hull property, the domain $D$ is relatively compact in $M_{0}$, then the image of $D$ under $f$ is automatically bounded, and the set of boundary values is simply the image under $f$ of the boundary of $D$. However, it is often useful to have the convex hull property in a more general setting, where $D$ need not be relatively compact. (In fact, $D$ may be all of $M$.) In that case, we use the following definitions.

A sequence of points in $D$ is divergent if no subsequence converges to a point of $D$.

The boundary values of $f(D)$ are all limit points of sequences $f\left(q_{n}\right)$ for all divergent sequences $\left\{q_{n}\right\}$ in $D$.

In the case that $D$ is relatively compact in $M$, divergent sequences in $D$ are precisely those which tend to the boundary of $D$, and the set of boundary values coincides with the image of the boundary.

5. If the convex hull property holds in the form stated, then it also holds in the following apparently stronger form: if $D$ is any domain on $M_{0}$ such that the sequence $\left\{f\left(q_{n}\right)\right\}$ is bounded for any divergent sequence $\left\{q_{n}\right\}$ in $D$, then the image of $D$ lies in the convex hull of its boundary values; namely, under these hypotheses, it follows easily that $f(D)$ is itself bounded.

In the proof of the theorem, we shall need the following lemma describing the local behavior of an immersion in terms of the normal curvatures.

Lemma. Let $p$ be a point of $M$, and $N$ be a unit normal vector to $M$ at $p$. Denote the principal curvatures of $M$ at $p$ with respect to $N$ by $k_{1}(N), \cdots$, $k_{m}(N)$ as above. For every $R>0$, denote by $B_{R}$ the closed ball of radius $R$ and center $c=p+R N$.

a) If some neighborhood of $p$ on $M$ lies in $B_{R}$, then $k_{m}(N) \geq 1 / R$.

b) If $k_{m}(N)>1 / R$, then some neighborhood of $p$ on $M$ lies in $B_{R}$.

c) If $k_{1}(N)>1 / R>k_{m}(N)$, then every punctured neighborhood of $p$ on $M$ contains points of $B_{R}$ and points outside $B_{R}$.

d) If $1 / R>k_{1}(N)$, then some punctured neighborhood of $p$ on $M$ lies outside of $B_{R}$.

Proof. Since the result is purely local, we may choose parameters $u_{1}, \cdots$, $u_{m}$ such that $M$ is given locally by a regular map $F(u)$ with $F(0)=p$. If we denote as usual

$$
g_{i j}=\frac{\partial F}{\partial u_{i}} \cdot \frac{\partial F}{\partial u_{j}},
$$


then we may further assume that $g_{i j}(0)=\delta_{i j}$. If $N$ is any unit normal to $M$ at $p$, then the principal curvatures $k_{1}(N), \cdots, k_{m}(N)$ are the eigenvalues of the matrix

$$
b_{i j}(N)=N \cdot \frac{\partial^{2} F}{\partial u_{i} \partial u_{j}}(0)
$$

Now with $c=p+R N$, we introduce the function

$$
h(u)=|F(u)-c|^{2} .
$$

Then

$$
\frac{\partial h}{\partial u_{i}}=2 \frac{\partial F}{\partial u_{i}} \cdot(F(u)-c)
$$

and

$$
\frac{\partial^{2} h}{\partial u_{i} \partial u_{j}}=2 \frac{\partial^{2} F}{\partial u_{i} \partial u_{j}} \cdot(F(u)-c)+2 \frac{\partial F}{\partial u_{i}} \cdot \frac{\partial F}{\partial u_{j}} .
$$

But $F(0)=p, h(0)=R^{2}$, and

$$
\begin{gathered}
\frac{\partial h}{\partial u_{i}}(0)=-2 R \frac{\partial F}{\partial u_{i}}(0) \cdot N=0 \\
\frac{\partial^{2} h}{\partial u_{i} \partial u_{j}}(0)=-2 R b_{i j}(N)+2 g_{i j}(0)=2\left(\delta_{i j}-R b_{i j}(N)\right) .
\end{gathered}
$$

Thus the matrix $\partial^{2} h / \partial u_{i} \partial u_{j}(0)$ has eigenvalues

$$
2\left(1-R k_{i}(N)\right), \quad i=1, \cdots, m .
$$

It follows that this matrix watrix will be

$$
\begin{aligned}
\text { positive definite } & \Longleftrightarrow k_{1}(N)<1 / R, \\
\text { negative definite } & \Longleftrightarrow k_{m}(N)>1 / R, \\
\text { negative semi-definite } & \Longleftrightarrow k_{m}(N) \geq 1 / R .
\end{aligned}
$$

Since $F(u)$ lies in $B_{R} \Longleftrightarrow h(u) \leq R^{2}$, the various cases in the lemma correspond to the sign of the function $h(u)-R^{2}$ near the origin. Analyzing this in the classical way in terms of the matrix of second derivatives (using Taylor's theorem) gives the result.

Remarks. 1. We shall actually only use parts (a) and (b) of the lemma.

2. No mention has been made of smoothness assumptions. What we need for the lemma is that $F \in C^{2}$, and hence that $f \in C^{2}$ in the theorem. 
Proof of the theorem. I. Suppose first that, at some point $p$ and for some unit normal $N$, all normal curvatures are positive, or, equivalently, $k_{m}(N)>0$. Choose $R>1 / k_{m}(N)$, and apply case (b) of the lemma. Let $p=f\left(q_{0}\right)$, and let $D$ be a neighborhood of $q_{0}$ whose image lies in the ball $B_{R}$. Since $f$ is an immersion, we may assume by further restricting $D$, if necessary, that $f$ is oneto-one on the closure of $D$. Then $f$ maps the boundary of $D$ onto a compact subset $E$ of $B_{R}-p$. On the set $E$, the function $(f(q)-p) . N$ has a positive minimum $\eta$. This means that the boundary values of $f(D)$ lie in the half-space $(x-p) \cdot N \geq \eta$, whereas $f(D)$ does not, since it contains $p$. Thus $M$ does not have the convex hull property.

II. Suppose, conversely, that $M$ does not have the convex hull property. Then there is some domain $D$ whose image is bounded but does not lie in the convex hull of its boundary values. Since the convex hull of a set is the intersection of all half-spaces in which it lies, there must exist a unit vector $v$ such that the half-space $x \cdot v \leq a$ contains the boundary values of $f(D)$, but not all of $f(D)$. This means that, for some $q_{0}$ in $D, f\left(q_{0}\right) \cdot v=b>a$.

We wish to show that, in this situation, there must be a point $p$ in $f(D)$ satisfying case (a) of the lemma. We may proceed as follows.

Let $B_{r}$ be a closed ball of radius $r$ and center $c_{r}$, where $c_{r} \cdot v=a$, such that the closure of $f(D)$ lies in the interior of $B_{r}$. For each $t>r$, we denote by $B_{t}$ the closed ball of radius $t$ and center $c_{t}=c_{r}-v \sqrt{t^{2}-r^{2}}$. Then the following statements are easily verified.

1. The boundary values of $f(D)$ lie in the interior of each $B_{t}$.

2. When $t$ is sufficiently large, the point $f\left(q_{0}\right) \notin B_{t}$.

3. For some value of $t$, say $t=R, f(D)$ lies in $B_{R}$, and some point $p$ of $f(D)$ lies on the boundary of $B_{R}$.

4. The tangent space to $M$ at $p$ is a subspace of the tangent space to the boundary of $B_{R}$; hence the unit vector $N$ directed from $p$ to $c_{R}$ is a normal vector to $M$ at $p$.

In view of these facts, case (a) of the lemma may be applied to conclude that all the normal curvatures of $M$ at $p$ with respect to $N$ are positive. This proves the theorem.

\section{Reference}

[1] R. Osserman, A survey of minimal surfaces, Van Nostrand Reinhold, New York, 1969. 